\title{
Characterization of the dnaK Locus in Streptomyces griseus
}

\author{
Kuninobu Inada ${ }^{1}$, Haruyasu Kinashi ${ }^{2}$, Osamu Nimi ${ }^{3}$, Toru Hasegawa ${ }^{4}$ and Hidenori Shinkawa ${ }^{2 *}$ \\ ${ }^{1}$ Department of Fermentation Technology, Graduate School of Engineering, Hiroshima University, Kagamiyama 1-4-1, \\ Higashi Hiroshima 739-8528, Japan \\ ${ }^{2}$ Department of Molecular Biotechnology, Graduate School of Advanced Sciences of Matter, Hiroshima University, Kagamiyama \\ 1-3-1, Higashi-Hiroshima 739-8530, Japan \\ ${ }^{3}$ Hiroshima Bunkyo Women's College, Kabehigashi 1-2-1, Asakitaku, Hiroshima 731-0295, Japan \\ ${ }^{4}$ Department of Food and Nutrition, Sonoda Women's University, Minamitsukaguchi 7-29-1, Amagasaki, 661-8520, Japan
}

(Received Nov. 6, 2002 / Accepted Dec. 4, 2002)

\begin{abstract}
The nucleotide sequence of the dnaK locus in Streptomyces griseus 2247 revealed a gene organization consisting of 6 genes (5'-orf1-dnaK-grpE-dnaJ-hspR-orf6-3'). The genes orf1 and orf6 were predicted to transcribe in the direction opposite that of $d n a K$, grpE, dnaJ and $h s p R$. This locus of $S$. griseus was compared with that of S. coelicolor A3(2). No homolog of orf1 and orf6 was found in S. coelicolor, while dnaK, grpE, $d n a J$ and $h s p R$ were highly similar to those of $S$. coelicolor. Promoter probing analysis revealed significant promoter activity in the upstream region of the $d n a K$ gene. The interspace regions of contiguous genes (dnaK-grpE-dnaJ-hspR) were amplified by RT-PCR of mRNA, indicating that these four genes constitute a polycistronic operon. His-tagged HspR protein was overexpressed in Escherichia coli and purified.
\end{abstract}

The synthesis of several proteins, known as the heat shock proteins (HSP), is rapidly induced when cells are exposed to stress conditions such as incubation at high temperature or the addition of alcohol to a medium. This phenomenon is commonly observed in a wide range of organisms. In Escherichia coli, DnaK, one of heat shock proteins, is involved in protein folding as a molecular chaperon co-operatively with GrpE and DnaJ ${ }^{1)}$. In Gramnegative bacteria, the expression of dnaK is positively regulated by RNA polymerase containing an alternative $\sigma$ factor, $\sigma^{32}$, which is a specific $\sigma$ subunit for heat-shock promoters $^{2)}$. In contrast, no homolog of $\sigma^{32}$ was found in Gram-positive bacteria. The expression of dnaK is regulated negatively by the HrcA/CIRCE system in Bacillus subtilis. HrcA (formerly called ORF39) binds to the specific sequence (CIRCE) in the dnaK promoter as a repressor ${ }^{3,4}$. We previously reported cloning and the nucleotide sequence of the dnaK ( $h s p 70)$ gene from Streptomyces griseus 22475). For further characterization of the dnaK locus in S. griseus, the flanking regions of dnaK were isolated and their nucleotide sequences were determined.

Nucleotide sequence of the dnaK locus in S. griseus and transcription analyses.

The nucleotide sequence of 6,214 bp dnaK locus in $S$. griseus revealed that six genes for possible open reading frames (ORF) were located in the order 5'-orf1-dnaKgrpE-dnaJ-hspR-orf6 -3' (Fig.1A). Both the nucleotide sequences and the predicted amino acid sequences of dnaK, grpE, dnaJ and $h s p R$ from $S$. griseus were highly similar to those of S. coelicolor A3(2). However, orfl and orf6 were not conserved in the S. coelicolor A3(2) genome ${ }^{6}$. From the results of DNA database searches, no homolog of ORF1 and ORF6 was found. The functions of orf1 and orf6 remain unclear. The genes orfl and orf6 were predicted to transcribe in the direction opposite that of dnaK, grpE, dnaJ and $h s p R$ (Fig.1A). A putative GTG initiation codon of GrpE (position 3,314) overlapped with the TGA termination codon of DnaK (position 3,315). The ORF of DnaJ started from the putative ATG initiation codon (position 4,061) at 116-bp downstream of the TGA termination codon of the GrpE. The putative ATG initiation codon of HspR (position 5,253) was located 1-bp behind the TGA termination codon of the DnaJ (position 5,249). The short interspace regions of the genes from dnaK through $h s p R$ appear to indicate that these 4 genes seemed to constitute an operon. For this reason, the promoter activity of the interspace regions shown in Fig.1A was examined. Promoter probing experiments using a promoter probe vector pML104 ${ }^{7)}$ showed significant promoter activity in the region upstream of $d n a K$. It was unclear whether promoters of dnaJ and $h s p R$ were present, although faint promoter activity was detected in the regions upstream of $d n a J$ and $h s p R$ due to the high-copy number plasmid used for promoter probing.

To determine the transcription unit of this region, RNA was prepared from mycelia treated for $20 \mathrm{~min}$. at $37^{\circ} \mathrm{C}$. Then, RNA was subjected to RT-PCR analyses using the primer sets shown Fig.1A. If they were transcribed from the dnaK promoter as a polycistronic mRNA, the interspace regions between $d n a K$ and grpE (region B), and between grpE and dnaJ (region C), between dnaJ and

* Corresponding author. Phone \& Fax: +81-824-24-7768. E-mail: hshinka@ hiroshima-u.ac.jp 
$h s p R$ (region D) were amplified. As shown in Fig.1B, all tested regions were amplified in the expected size, except the region upstream of $d n a K$ (region A). These amplified signals disappeared when RNA treated with RNase was used for RT-PCR. These amplified bands were not found in a PCR experiment of the same RNA sample without reverse transcriptase. An approximately $4.3-\mathrm{kb}$ transcript, which corresponded to a polycistronic mRNA of dnaK, grpE, dnaJ and $h s p R$, was detected by all northern blot analyses using the specific probes for dnaK, grpE, dnaJ and $h \operatorname{spR}$ (data not shown). As expected, the region upstream of $d n a K$ (region A) was not amplified. The $h s p R$ gene was followed by an inverted repeat that may form a stem-loop structure (position 5,737 to 5,780). This potential structure may function as a major terminator. The orf6 located downstream of $h s p R$ was predicted to transcribe in the direction opposite that of $h s p R$. Both genes appeared to share the terminator. On this basis, we concluded that the genes from $d n a K$ through $h s p R$ were transcribed from the $d n a K$ promoter as a polycistronic operon.

\section{DNA-binding ability and effect on transcription of HspR protein.}

An ORF of $h s p R$ consisted of 150 amino acid residues. The deduced amino acid sequence of the $S$. griseus HspR protein was highly similar to the $S$. coelicolor A3(2) HspR, the GlnR repressor proteins of Bacillus species and mem- bers of the MerR family of eubacterial transcription regulators ${ }^{8)}$. A helix-turn-helix DNA binding motif was found in the amino terminus region of HspR. HspR appeared to be involved in the regulation of the dnaK promoter. To examine DNA binding ability, S. griseus HspR protein was overexpressed in E. coli and purified from the cell extract. The specific binding of HspR to the dnaK promoter was indicated by a gel shift assay (Fig.2A). The retarded mobility of DNA fragment was generated by adding HspR. In competition experiment, adding a cold probe eliminated the gel mobility shift caused by HspR binding, thereby identifying the specific binding of HspR to the dnaK promoter. As demonstrated previously, the dnaK promoter was recognized by RNA polymerase holoenzyme purified from vegetative-stage mycelia of S. griseus $^{9)}$, and HspR of $S$. coelicolor A3(2) played as the role of repressor ${ }^{10)}$. We therefore tried to test in vitro run-off transcription using a RNA polymerase holoenzyme prepared from $S$. griseus in the presence of HspR (Fig.2B). The expression of the dnaK promoter was decreased in accordance with increasing amounts of HspR. These results indicated that HspR binds to the dnaK promoter to repress the transcription of dnaK operon. However, repression in vitro with $\mathrm{HspR}$ appeared incomplete, because an excess amount of HspR to template DNA was required in a run-off transcription experiment. The results suggest two possibilities:1) that the folding of the recombinant HspR protein is incomplete,
A
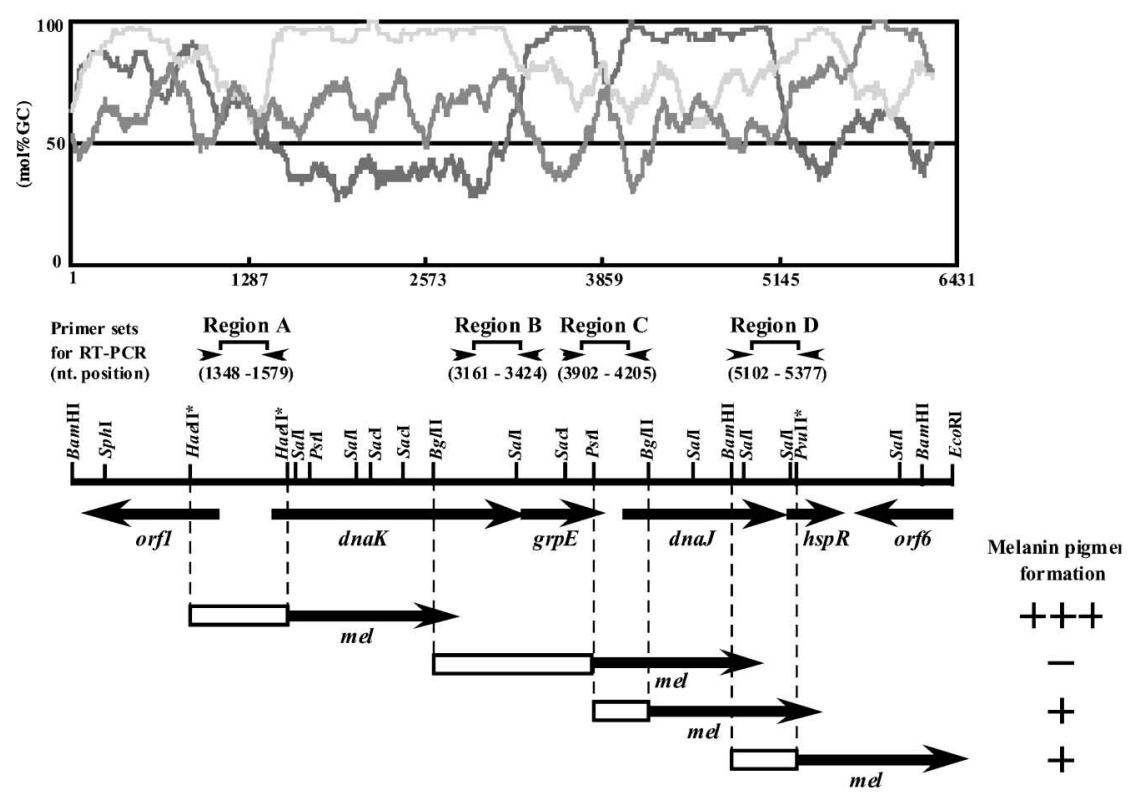

\section{B}

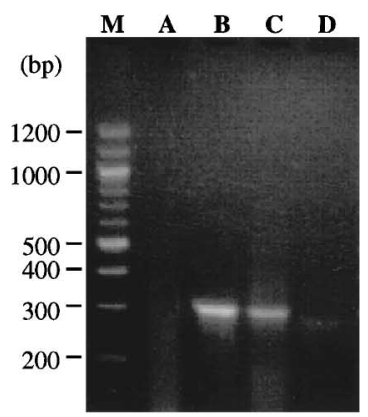

Fig.1. Physical map of the dnaK locus in S. griseus 2247, promoter probing and RT-PCR analysis.

(A) Thick horizontal arrows below the restriction map of the sequenced region represent the location and transcriptional direction of the ORFs predicted by frame analysis. The frame analysis result is shown above the restriction map. The nucleotide sequence has been deposited in the DDBJ/EMBL/GenBank under the accession number D14499. Constructs for promoter probing are indicated below the restriction map by thick arrows with open boxes. Melanin pigment production represents promoter activity. (B) RT-PCR analysis was carried out using the primer (24 mer) sets shown in (A) and an RTPCR high kit (Toyobo, Co.) according to the provider's manual except for annealing temperature $\left(60{ }^{\circ} \mathrm{C}\right)$. RNA was prepared by the previous method. Lane M represents 100 bp ladder as a marker. Lanes A-D correspond to the regions shown in (A) 
A

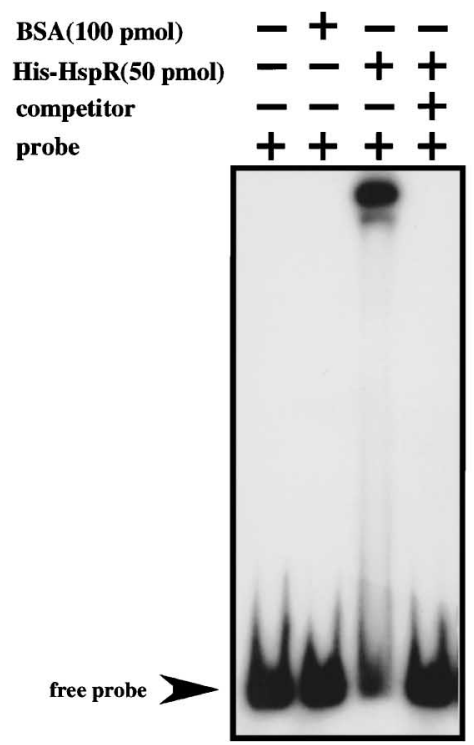

B

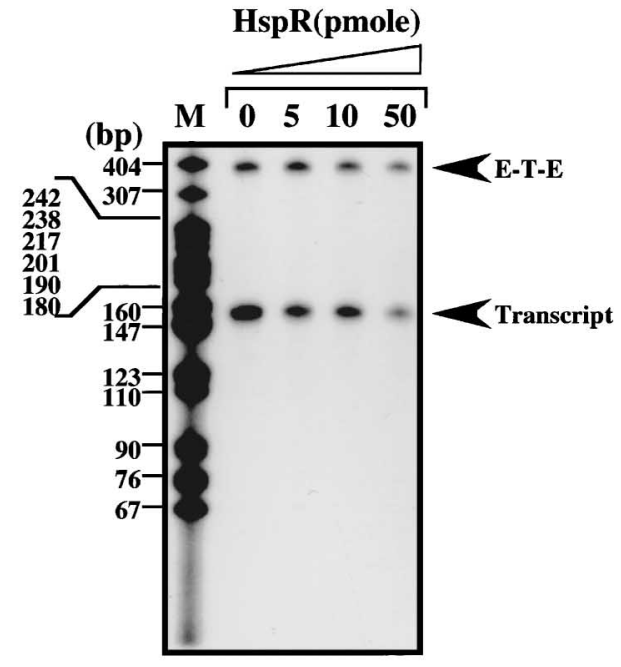

Fig.2. Gel shift assay and in vitro transcription of dnaK promoter with HspR.

(A) HspR protein was overexpressed in E. coli BL21(DE3) using the pET vector system. His-tagged HspR protein was purified by chromatography through $\mathrm{Ni}^{2+}$-nitrilotriacetic acid column. Gel shift assay was performed by the method of Retzlaff \& Distler ${ }^{12)}$ using a 150-bp DNA fragment of the dnaK promoter (nt. 1338-1488). The composition of the reaction mixture is indicated at the top of the gel. (B) In vitro transcription experiment was carried out by the previous method 9) using a 340-bp DNA fragment of the dnaK promoter (nt. 1177-1517). The amount of HspR added to reaction mixture is indicated at the top of the gel. E-T-E represents an end-to-end product of the template used. Lane M represents ${ }^{32} \mathrm{P}$-labeled Msp I-fragments of pBR322 as a marker.

or 2) that HspR requires another co-operative factor(s). DnaK has been reported to enhance the function of HspR as a repressor of the dnaK operon in S. coelicolor A3(2) ${ }^{11)}$. The same system may be conserved in S. griseus.

In $S$. griseus, the flanking regions of dnaK operon differed from the corresponding regions of $S$. coelicolor $\mathrm{A} 3(2)$, while the gene organization of the dnaK operon and function of HspR were conserved. This suggests that the chromosomal location of the dnaK operon in S. griseus differs from $S$. coelicolor A3(2). The completion of genome sequencing of $S$. griseus, should clarify this issue.

\section{REFERENCES}

1) Liberec, K., Marszalek, J., Ang, D., Georgopoulos, C. \& Zylicz, M.: Escherichia coli DnaJ and GrpE heat shock proteins jointly stimulate ATPase activity of DnaK. Proc. Natl. Acad. Sci. USA, 88: 2874-2878, 1991.

2) Yura, T., Nagai, H. \& Mori, H.: Regulation of heat shock response in bacteria. Annu. Rev. Microbiol. 47: 321-350, 1993.

3) Zuber, U. \& Schumann, W.: CIRCE, a novel heat shock element involved in regulation of heat shock operon dnaK of Bacillus subtilis. J. Bacteriol., 176: 1359-1363, 1994.

4) Yuan, G. \& Wong, S.: Isolation and characterization of Bacillus subtilis groE regulatory mutants: evidence for orf39 in the $d n a K$ operon as a repressor gene in regulating the expression of both groE and dnaK. J. Bacteriol., 177: 6462-6468, 1995.
5) Hatada, Y., Shinkawa, H., Kawamoto, K., Kinashi, H. \& Nimi, O.: Cloning and nucleotide sequence of a $h s p 70$ gene from Streptomyces griseus. J. Ferment. Bioeng., 77: 461-467, 1994.

6) Bentley, S.D., Chater, K.F, Cerdeño-Tárraga, A.-M., Challis, G.L., Thomson, N.R., James, K.D., Harris, D.E., Quail, M.A., Kieser, H., Harper, D., Bateman, A., Brown, S., Chandra, G., Chen, C.W., Collins, M., Cronin, A., Fraser, A., Goble, A., Hidalgo, J., Hornsby, T., Howarth, S., Huang, C.-H., Kieser, T., Larke, L., Murphy, L., Oliver, K., O’Nell, S., Rabbinowitsch, E., Rajandream, M.-A., Rutherford, K., Rutter, S., Seeger, K., Saunders, D., Sharp, S., Squares, R., Squares, S., Taylor, K., Warren, T., Wietzorrek, A., Woodward, J., Barrell, B.G., Parkhill, J. \& Hopwood, D.A.: Complete genome sequence of the model actinomycete Streptomyces coelicolor A3(2). Nature, 417: 141-147, 2002.

7) Shinkawa, H., Hatada, Y., Okada, M., Kinashi, H. \& Nimi, O.: Nucleotide sequence of a principal sigma factor gene (hrdB) of Streptomyces griseus. J. Biochem., 118: 494-499, 1995.

8) Bucca, G., Ferina, G., Puglia, A. M. \& Smith, C.P.: The dnaK operon of Streptomyces coelicolor encodes a novel heat-shock protein which binds to the promoter region of the operon. Mol. Microbiol., 17: 663-674, 1995.

9) Shinkawa, H., Fujita, N., Shiina, T., Tanaka, K., Takahashi, H., Ishihama, A. \& Nimi, O.: Purification and characterization of RNA polymerase holoenzyme $\left(E \sigma^{\mathrm{B}}\right)$ from vegetativephase mycelia of Streptomyces griseus. J. Biochem., 118: 488-493, 1995. 
10) Bucca, G., Hindle, Z. \& Smith, C.P.: Regulation of the dnaK operon of Streptomyces coelicolor A3(2) is governed by HspR, an autoregulatory repressor protein. J. Bacteriol., 179: 5999-6004, 1997.

11) Bucca, G., Brassington, A.M.E., Schönfeld, H.-J. \& Smith, C.P.: The HspR regulon of Streptomyces coelicolor: a role for the DnaK chaperone as a transcriptional co-repressor. Mol. Microbiol., 38: 1093-1103, 2000.

12) Retzlaff, L. \& Distler, J.: The regulator of streptomycin gene expression, StrR, of Streptomyces griseus is a DNA binding activator protein with multiple recognition sites. Mol. Microbiol., 18: 151-162, 1995. 